\title{
Formación emocional del profesorado y gestión del clima de su aula
}

Amelia Barrientos Fernández

Doctora Educación

Universidad Alfonso X el Sabio - España abarrien@uax.es

https://orcid.org/oooo-0oo3-4185-243X

\section{Roberto Sánchez Cabrero}

Doctor. Psicología

Universidad Alfonso X el Sabio - España rcabrero@uax.es

https://orcid.org/oooo-0002-1978-7531

\section{Amaya Arigita García}

Doctora. Ciencias Sociales y Educación Universidad Alfonso X el Sabio - España aarigita@uax.es

https://orcid.org/oooo-0oo3-2965-7252

\section{Artículo de investigación}

Recepción: 14 de abril de 2019

Aprobación: 30 de julio de 2019

https://doi.org/10.19053/22160159.v10.n25.2019.9894

\section{Resumen}

Este artículo de investigación tiene como punto de partida el paradigma educativo y de mentalidad en la sociedad actual, en el que el profesorado se ha visto en la necesidad de desarrollar habilidades -también emocionales y sociales- que le ayuden a gestionar sus clases de forma positiva. El objetivo general del estudio es conocer la relación entre la formación en inteligencia emocional, que deben poseer los docentes, y su capacidad para gestionar el clima emocional y social del aula. La metodología de investigación es de tipo descriptivo. Los resultados muestran que el profesorado tiene la percepción de que no ha sido instruido en aptitudes emocionales y, por ello, siente la necesidad de ser formado en habilidades socioemocionales para poder gestionar las clases de forma positiva.

Palabras clave: formación del profesorado, inteligencia emocional, habilidad emocional, habilidad social 


\title{
Teachers' emotional training and management of their classroom atmosphere
}

\begin{abstract}
This research article has as its starting point the educational and mentality paradigm in today's society, in which teachers have been in need of developing skills. The overall objective of the study is to know the relationship between the emotional intelligencetraining that teachers should have-and their ability to manage the emotional and social climate of the classroom. The research methodology is descriptive. The results show that teachers perceive that they have not been instructed in emotional skills and, therefore, they feel the need to be trained in social-emotional skills in order to be able to manage classes in a positive way.
\end{abstract}

Keywords: teacher training, emotional intelligence, emotional ability, social ability

\section{La formation émotionnelle des enseignants et la gestion de l'environnement de leurs salles de classe}

\section{Résumé}

En raison du changement de paradigme éducatif et des mentalités, les enseignants ont dû développer des compétences aussi bien émotionnelles que sociales, leur permettant de mieux exécuter leurs cours. L'objectif général de cette étude est de déterminer la relation entre la formation sur l'intelligence émotionnelle que les 
enseignants doivent avoir et leur capacité à gérer l'environnement émotionnel et social de la classe. La méthode de recherche utilisée est de type descriptif. Les résultats montrent que les enseignants considèrent que leur formation n'aborde pas les capacités émotionnelles et, par conséquent, ils éprouvent le besoin d'être formés au développement de compétences socio-émotionnelles pour mieux exécuter leurs cours.

Mots-clés : formation des enseignants, intelligence émotionnelle, compétence émotionnelle, compétence sociale

\section{Formação emocional do professorado e gestão do clima de sua sala}

\section{Resumo}

Devido à mudança do paradigma educativo e de mentalidade na sociedade atual, o professorado tem-se visto na necessidade de desenvolver habilidades - também emocionais e sociais - que lhe ajudem a administrar suas classes de forma positiva. O objetivo geral do estudo é conhecer a relação entre a formação em inteligência emocional, que devem possuir os docentes, e sua capacidade para administrar o clima emocional e social da sala. A metodologia de pesquisa é de tipo descritivo. Os resultados mostram que o professorado tem a percepção de que não têm sido instruídos em aptidões emocionais e, por isso, sente a necessidade de ser formado em habilidades sócio emocionais para poder administrar as classes de forma positiva.

Palavras-chave: formação do professorado, inteligência emocional, habilidade emocional, habilidade social 


\section{Introducción}

En la actualidad, muchos docentes están desconcertados por el comportamiento y actitud que muestran sus alumnos en las aulas. Comprueban, a pesar de estar convencidos de ello, que no solo las estrategias no funcionan de igual forma que hace unos años, sino que ya no sirve el manejo tradicional de sus aulas. Deben plantearse poner en práctica destrezas de enseñanza adaptadas a la manera de aprender del alumnado en todas sus dimensiones - cognitiva, social, moral, emocional, entre otras- (Pérez-Escoda, Filella, Alegre \& Bisquerra, 2012). Los maestros se sienten sobrepasados y desconcertados con los cambios sociales habidos en los últimos años que están afectando a todos y, en especial, a sus alumnos, por lo que creen que es necesario abordar las dificultades que surjan entre la escuela y la familia (Retamal \& González, 2019).

En este siglo, ser docente es una labor cada vez más complicada. Ello ha dado lugar a la concienciación de que los docentes deben formarse a nivel emocional y social (Berrocal, Cabello \& Cobo, 2017), lo cual constituye, por tanto, una labor indispensable que conllevaría una gran ventaja para su desempeño profesional, tanto a nivel intrapersonal como interpersonal (Cassullo \& Labandal, 2015). Para ello, la sociedad empieza a poner en práctica programas de formación en inteligencia emocional. Además, numerosos colegios están introduciendo educación emocional en su programación general anual, no solo para alcanzar un buen crecimiento a nivel personal y social, sino también para lograr una buena convivencia en el centro y lograr un bienestar general para el alumnado (PérezEscoda et al., 2012).

La capacidad para comportarse de forma apropiada y de relacionarse bien con los demás se aprende en el ambiente familiarescolar y, para que sea efectivo el aprendizaje, debe haber una colaboración entre ambos contextos, que facilite la convivencia y el respeto de los valores enseñados, como son la autonomía, la responsabilidad y el esfuerzo (Bisquerra, Pérez-González \& GarcíaNavarro, 2015).

Para dotar a los docentes de las habilidades necesarias para manejar de forma adecuada sus grupos de alumnos, se hace necesaria una formación específica en educación emocional y, para ello, los colegios están cada vez más interesados en contar con 
profesionales formados para instruir a sus profesores. Además, los padres y docentes están preocupados por formar a sus alumnos en inteligencia emocional con el fin de que desarrollen estrategias que les habiliten para manejar su vida emocional y hacerse responsables de sus acciones y comportamientos (Martínez, Rodríguez, Álvarez \& Becedóniz, 2016).

Robira y Bris (2012) opinan que los maestros deben contar con recursos suficientes para lidiar con la tensión, no solo con la que se encuentran en sus clases, sino también la de gestionar las relaciones con sus estudiantes, con otros profesionales y con los padres. Para este fin, cuentan con la posibilidad de usar las emociones positivas que les permitan alcanzar su bienestar emocional, lo cual influye de forma positiva en su desarrollo racional y en su comportamiento. Todo ello redundará en una mejora de la capacidad para manejar el clima del aula y, en consecuencia, en mejorar las relaciones alumno-profesor.

Sin embargo, para Ulloa, Evans y Jones (2016), hay escasa formación en competencias emocionales y sociales que reciben, no solo los futuros profesores, sino también los profesionales que, aun ejerciendo, necesitan de esta formación para gestionar adecuadamente el clima de sus aulas. Ellos reclaman, por tanto, ser entrenados en competencias tales como la empatía, asertividad, autoestima, el control del estrés y la capacidad de ser flexibles.

En este sentido, se han puesto en marcha desde hace algunos años programas de educación emocional, tanto dentro como fuera de los centros, pero en general son cursos muy teóricos y no enseñan a los docentes a poner en práctica sus contenidos. Por esta razón, es importante que se adecúen a la realidad y que permitan a los profesores aplicarlos en las clases (Gordillo, Ruiz, Sánchez \& Calzado, 2016).

Asimismo, sería aconsejable incluir en la formación del profesorado, tanto de forma inicial como continua, cursos con contenidos de índole socioemocional, para que los maestros se encuentren más capacitados y preparados para ocuparse de la educación de los alumnos. En este sentido, Gordillo y SánchezHerrera (2015) constataron que los estudiantes de carreras de modalidad pedagógica a lo largo de los cursos adquieren y mejoran sus habilidades sociales y emocionales. 
Además, los docentes que son capaces de reconocer, comprender y regular sus propias emociones se suelen sentir más competentes para enseñar a sus discentes a manejar su vida emocional de manera adecuada (Burrola-Herrera, Burrola-Márquez\&Viramontes-Anaya, 2016). En relación con lo anterior, cada vez son más los directores de centros educativos que están preocupados por incluir en sus centros programas en formación emocional, y así proporcionar a sus profesores medios para manejar el clima emocional de su aula y favorecer también las relaciones entre ellos, con sus alumnos y entre alumnos (Bisquerra \& Hernández-Paniello, 2017).

Caruana (2013) sostiene que los padres y profesores deben educar emocionalmente a los niños desde la infancia con el fin de prepararlos para el futuro y capacitarlos para enfrentarse a posibles problemas, y mejorar así su estado personal y su capacidad para relacionarse con los demás (Pérez-Escoda et al, 2012).

En muchas ocasiones los profesores tienen que enfrentarse a circunstancias difíciles. Para ello deben contar con estrategias adecuadas con el fin de mantener la disciplina en sus clases, ya que deben ser capaces de convertirse en buenos líderes y ser respetados. Se hace esencial, por lo tanto, la consecución de habilidades que les faciliten la organización de sus aulas, y más aun si estas son de carácter inclusivo (Ros-Morente, Filella, Ribes \& Pérez-Escoda, 2017).

Muchos son los profesores que en determinados momentos están en la obligación de abordar dificultades emocionales por parte de sus alumnos, como puede ser la separación de sus padres, problemas de desempleo en sus familias, falta de atención familiar por el trabajo de los padres, entre otros. Para ello, proporcionar a los alumnos herramientas útiles para hacer frente a dichas circunstancias y crear un ambiente emocionalmente positivo en el aula, ayudará a formar al alumno como una persona íntegra en todas sus competencias (Gordillo et al., 2016).

Las competencias que se consideran necesarias para los maestros y que deben ser capaces de poner en práctica son: saber amoldarse a situaciones inesperadas en sus clases, relacionarse de forma empática con sus alumnos, trabajar en grupo de forma colaborativa, ser autónomos, saber abordar situaciones estresantes, ser emprendedores, ser líderes, ser capaces de motivar a sus alumnos y, sobre todo, ser asertivos (Pérez-Escoda et al, 2012). 
Bisquerra y Pérez-Escoda (2007) afirman que, a pesar de que se están llevando a cabo programas de educación emocional en las escuelas, el profesorado no está suficientemente formado. Por ello insisten en esta necesidad. Por otro lado, los directores de los centros educativos y los maestros, en general, están concienciados y sensibilizados de la necesidad de incluir dentro de sus escuelas programas curriculares de contenido emocional, pero encuentran dificultades para hacerlo, debido a que se sienten incapaces por no haber sido entrenados para ello y, por lo tanto, no se sienten capaces de enseñar lo que no saben (Bisquerra \& HernándezPaniello, 2017).

Gordillo y Sánchez-Herrera (2015) se preguntan si realmente los estudiantes de las distintas facultades de educación están adquiriendo las competencias emocionales necesarias para su formación como docentes. En su estudio concluyen que ha habido una alta mejoría en su formación, a pesar de que sigue siendo precaria y teórica.

Por último, para que la educación emocional del profesorado sea efectiva es clave que se realicen programas de capacitación ratificados científicamente y que tengan consistencia teórica, que cuenten con una planificación apropiada para su aplicación práctica y que impliquen a la mayoría del colectivo educativo (Durlak, 2016; García \& Sánchez, 2017; Martínez-Izaguirre, Yániz \& Villardón, 2017). En muchos de los programas de formación emocional se están creando contenidos teórico-prácticos -dinámicas de grupo, escenificaciones, actividades dialógicas, entre otras - y actividades que están destinadas a mejorar las competencias sociales y emocionales del profesorado de todas las etapas (Rueda \& Filella, 2016).

\section{Objetivos}

El objetivo general del presente trabajo de investigación es describir el rendimiento en gestión del aula, en relación con el apoyo emocional, de los docentes de educación infantil en el marco educativo español. Para ello, será necesario reflejar adecuadamente la influencia de tres variables que podrían ser relevantes para la gestión emocional del aula. Dichas variables son: la titularidad del centro educativo, el número de alumnos en el aula y haber recibido formación en aspectos socioemocionales previamente, o no. 
Los objetivos específicos considerados son:

- Describir la calidad del apoyo emocional del docente en su aula, tanto de forma global como teniendo en cuenta la titularidad del centro educativo y haber recibido formación en aspectos socioemocionales previamente.

- Determinar si la titularidad del centro educativo y haber recibido formación en aspectos socioemocionales previamente son factores significativos en relación con la calidad del apoyo emocional del docente en las aulas de educación infantil.

- Valorar si existe una correlación significativa entre el número de alumnos y la capacidad de los maestros para gestionar el clima del aula.

\section{Método de investigación}

\section{Población y muestra}

En este estudio se analiza directamente la formación y habilidades para la gestión del aula de los profesores de alumnos en edad temprana -menores de 6 años-, correspondientes a la etapa de educación infantil del marco educativo español. Para describir adecuadamente dicha población y poder extrapolar las conclusiones obtenidas, se ha utilizado una muestra de 68 profesores de educación infantil de centros educativos de la zona oeste de la Comunidad de Madrid, España. Los datos que se han obtenido pertenecen a 68 aulas de segundo ciclo de educación infantil -alumnos de 3 a 6 años-que cuentan con un total de 1493 alumnos y 68 profesores, de los cuales $61-89,71 \%$ - son maestras y $7-10,29 \%-$ son maestros.

Se realizó inicialmente un muestreo por conglomerados para facilitar la recogida de datos mediante abordaje etnográfico. Una vez seleccionados los centros educativos donde realizar el estudio, la selección de las aulas se ha llevado a cabo de forma aleatoria en $22-32,4 \%$ - centros privados, en $26-38,2 \%$ - centros concertados y en $20-29,4 \%-$ centros públicos, para facilitar que la heterogeneidad de la muestra describa adecuadamente la variabilidad inherente de la población de referencia y garantizar la validez ecológica de los resultados. De las 68 clases, $25-36,8 \%-$ pertenecen al primer curso de educación infantil -alumnos de 3 
años-; $21-30,9 \%$ - al segundo curso - alumnos de 4 años - ; y 22 $-32,4 \%$ - al tercer curso - alumnos de 5 años-.

\section{Variables evaluadas e instrumentos de recogida de datos}

En este estudio se mide el apoyo emocional proporcionado por el docente a sus alumnos en el aula mediante observación sistemática en un abordaje etnográfico. Se evalúan las diferencias existentes sobre el apoyo emocional según la variabilidad inherente en tres variables atributivas previamente consideradas.

La primera variable es la titularidad del centro educativo. Se trata de una variable nominal con tres condiciones: centros públicos, privados o concertados.

La segunda variable es docentes formados previamente en formación socioemocional. Se trata de una variable nominal dicotómica con dos condiciones: sí recibió formación previa y no la ha recibido.

La última de las variables es número de alumnos por aula y es cuantitativa discreta. Hace referencia al número de alumnos presentes en el aula durante la medición del CLASS-K mediante abordaje etnográfico.

Se han recogido datos respecto a si los profesores en sus centros educativos habían recibido algún curso de educación emocional en el que se les haya formado o no en habilidades emocionales interpersonales e intrapersonales-, a través de una consulta directa con un cuestionario básico de carácter general.

Para la obtención de información sobre el apoyo emocional del profesorado en sus clases se ha usado el cuestionario CLASS-K (Pianta, La Paro \& Hamre, 2008), ya que se trata de un test baremado y validado, que fue adaptado a la población hispanohablante por Treviño (2013) en aulas de educación infantil -niños de 3 a 6 añosde Chile, con un coeficiente de confiabilidad de 0,94. Cuenta con una consistencia muy alta de interevaluadores $-\alpha$ de Cronbach: 0,78 a 0,96- y una también muy alta confiabilidad interna $-\alpha$ de Cronbach: 0,78 a 0,90-. El cuestionario mide tres dimensiones: a) el manejo emocional o la habilidad del maestro para gestionar el clima socioemocional del aula; b) la organización de la clase, que consiste en la capacidad del profesor para gestionar las conductas o comportamientos de sus alumnos y de programar el trabajo y las 
actividades didácticas; $y$, por último, c) el apoyo pedagógico, que es la habilidad del docente para fomentar en sus estudiantes aptitudes lingüísticas y de pensamiento. Sin embargo, en esta investigación solo se ha evaluado la primera dimensión del cuestionario, es decir, el manejo o apoyo emocional, que incluye las siguientes subdimensiones: clima positivo y negativo, sensibilidad del profesor para estar atento a las necesidades emocionales y académicas de sus alumnos, la atención del docente a las motivaciones de sus alumnos y la capacidad de darles libertad para aprender de forma autónoma.

Para la recogida de datos se ha utilizado la técnica de observación sistemática mediante abordaje etnográfico; se intenta describir la realidad de las aulas, afectando lo menos posible el funcionamiento real de cada aula. Para garantizar dicho logro, se ha contado con dos observadores que previamente han sido formados e instruidos, con el fin de adecuarse a los criterios de rigor sistemático.

\section{Procedimiento}

Antes de comenzar el estudio se obtuvo consentimiento escrito informado de los directores de los centros, a través de una carta formal en la que se recogían los detalles de la investigación. Ellos obtuvieron consentimiento escrito informado de los padres de los alumnos para realizar las observaciones de las actividades de sus hijos en las aulas. En todo momento se garantizó el anonimato de los participantes y no se realizó intervención alguna que pudiera afectar a los alumnos o al funcionamiento habitual de cada aula.

El estudio consistió en la observación por parte de dos observadores en tres momentos diferentes - de 20 minutos cada uno- dentro de la jornada escolar, en los que los niños estaban haciendo actividades educativas diversas.

\section{Diseño de investigación}

La investigación es de tipo descriptivo y correlacional, ya que se hace una descripción del comportamiento de las variables del estudio sin realizar intervención alguna o modificar la realidad estudiada y se valora la relación existente entre las competencias sociales y emocionales de los maestros, su experiencia previa en formación socioemocional, el ratio de alumnos por aula y la forma de gestionar el clima de sus aulas. 
Para la obtención de los datos se hicieron dos exploraciones: en primer lugar, la relacionada con las variables generales, que incluyen aspectos sobre el número de alumnos por aula y los cursos de formación socioemocional recibidos por los profesores; en segundo lugar, para conocer el apoyo emocional de los maestros en sus clases, se hizo un análisis a través de la técnica de observación sistemática, llevada a cabo por dos observadores, en tres momentos diferentes a lo largo del día, en los que el profesor llevaba a cabo distintas actividades didácticas con sus alumnos.

\section{Modelo de análisis de datos}

Los análisis estadísticos inferenciales y correlacionales fueron realizados con el paquete informático estadístico SPSS, versión 18.o, y con el de Microsoft Excel del paquete Office de Microsoft. Para el tratamiento estadístico de los datos se categorizaron las variables y se hicieron los siguientes análisis:

- Análisis estadísticos de frecuencias y descriptivos, para conocer y describir el funcionamiento de las variables estudiadas en la muestra. Entre los análisis se incluyen las frecuencias, los porcentajes y las medias aritméticas de la titularidad de los centros de la muestra, el número de alumnos por clases y los profesores que han recibido o no cursos de formación emocional.

- Análisis de correlación $-r$ de Pearson- para conocer la correlación entre el número de alumnos por clase y la habilidad del profesor para gestionar el clima de sus aulas.

- $\quad$ ANOVA de un factor, mediante $F$ de Snedecor, que establece la significación estadística de la relación entre las medias de los profesores de las distintas titularidades del centro y la relevancia de la formación previa en inteligencia emocional con su capacidad para manejar el clima de sus aulas.

\section{Interpretación de resultados}

En la tabla 1 se muestra la distribución de la muestra según las variables consideradas y los resultados sobre la normalidad de distribución de los datos a través de la asimetría y curtosis. 


\section{Tabla 1}

Distribución de frecuencias de las variables descriptivas de la muestra y evaluación de la normalidad de la distribución de los datos

\begin{tabular}{ccccc}
\hline & Frecuencia & Porcentaje & Asimetría & Curtosis \\
\cline { 2 - 5 } & & & & \\
Titularidad del centro educativo & 20 & $29.4 \%$ & -.053 & -1.390 \\
Público & 26 & $38.2 \%$ & & \\
Concertado & 22 & $32.4 \%$ & & \\
Privado & & & & \\
$\quad$ & & & -.367 & -1.923 \\
Docentes formados previamente & 28 & $41.2 \%$ & & \\
en formación socioemocional & 40 & $58.8 \%$ & & \\
Sí & 68 & $100 \%$ & & \\
No & & & & \\
Total de centros & & & -.296 & .548 \\
$\quad$ Número de alumnos por aula & 17 & $25 \%$ & & \\
$<$ de 20 & 42 & $61.8 \%$ & & \\
De 20 a 25 & 9 & $13.2 \%$ & & \\
$>$ de 25 & 68 & $100 \%$ & & \\
Total de alumnos 1.493 alumnos & & & & \\
\hline
\end{tabular}

Fuente: elaboración propia.

Como puede observarse, la distribución de la tipología de los centros educativos ha sido bastante equitativa, ya que el 30\% son públicos, el $38 \%$ concertados y el $32 \%$ privados. Asimismo, se indica que el número total de alumnos de la muestra es de 1493, que están distribuidos en 68 aulas, cuya media aritmética por aula es de $X$ : 21.96 con una distribución típica de $d t: 3.671$ alumnos. Este dato es positivo porque la ratio de estudiantes por profesor se encuentra entre 20 y 25. De las 68 aulas evaluadas, solo 7 se alejan bastante de la media y los 61 restantes están alrededor de dicha media.

En relación con los resultados sobre la formación emocional de los profesores, podemos observar que es significativo el porcentaje de profesorado $-59 \%$ - que responde que no ha sido instruido en educación emocional. Es destacable el dato de que menos de la mitad -41\% - de los profesores responden sí haber sido formados.

Finalmente, los datos de asimetría y curtosis reflejan una distribución normal de los datos, por lo que es recomendable hacer análisis estadísticos inferenciales paramétricos en este estudio. Se puede tomar como muestra representativa el histograma de 
la figura 1, donde puede observarse gráficamente la uniformidad de la distribución del número de alumnos por aula de la muestra estudiada y, por tanto, ver con claridad cómo sus datos siguen una distribución normal.

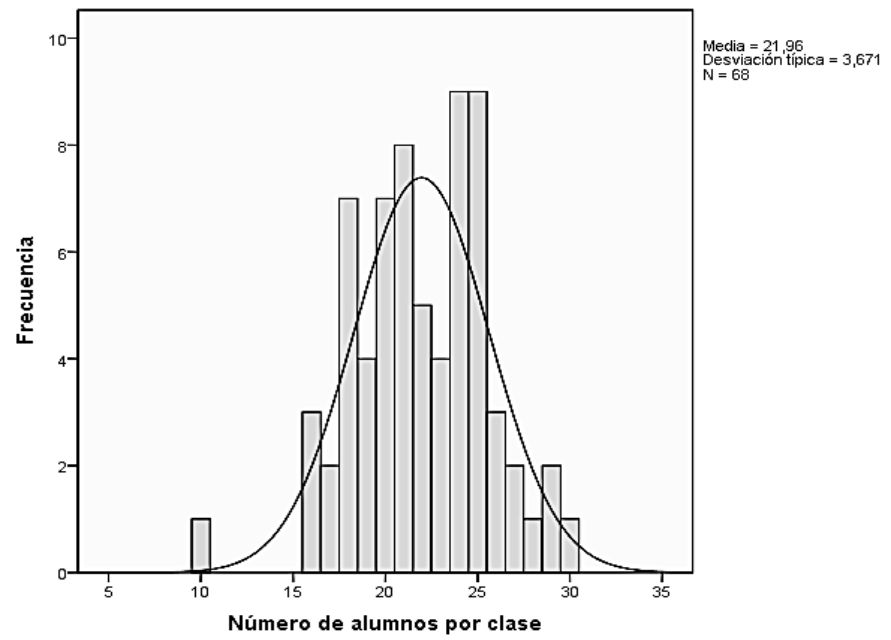

Figura 1. Histograma de número de alumnos por aula.

En la tabla 2, pueden observarse las medias aritméticas y desviaciones típicas obtenidas a través de la observación sistemática realizada con el $C L A S S-K$, tanto los resultados directos como los filtrados según las tres variables atributivas consideradas.

Tabla 2

Medias aritméticas y desviaciones típicas obtenidas en apoyo emocional a través de la observación sistemática realizada con el CLASS-K

\begin{tabular}{cccc}
\hline & $\boldsymbol{N}$ & $\boldsymbol{X}$ & $\boldsymbol{d t}$ \\
\cline { 2 - 4 } Titularidad del centro educativo & 20 & 16,08 & 2,774 \\
$\quad$ Público & 26 & 17,68 & 3,215 \\
$\quad \begin{array}{l}\text { Concertado } \\
\text { Privado }\end{array}$ & 22 & 18,82 & 2,579 \\
Docentes formados previamente en formación socioemocional & & & \\
Sí & 28 & 16,71 & 3,552 \\
No & 40 & 18,18 & 2,516 \\
& 68 & & \\
\hline
\end{tabular}

Fuente: elaboración propia. 
En la tabla 2, se observa que en los centros privados la media del manejo emocional de los profesores es ligeramente superior a la de docentes de los centros públicos y concertados. Por tanto, la titularidad - pública, concertada o privada- no influye en la habilidad de los docentes para apoyar social y emocionalmente a sus alumnos y para crear un clima adecuado que favorezca unas buenas relaciones entre sus miembros y cree unas condiciones apropiadas para el desarrollo de los alumnos.

En relación con los análisis estadísticos inferenciales, teniendo en cuenta que de las tres variables intervinientes consideradas una de ellas es cuantitativa -número de alumnos por aula-, se realizarán dos tipos de análisis: por un lado, un análisis de correlación existente entre la observación sistemática del apoyo emocional medida con el CLASS-K y el número de alumnos por aula, mediante $r$ de Pearson: y, por otro lado, un contraste de diferencia de medias mediante la prueba $t$ de Student, que relaciona los resultados de la observación sistemática de los docentes, teniendo en cuenta las distintas condiciones consideradas en las variables nominales categóricas de titularidad del centro educativo y docentes formados previamente en formación socioemocional.

Respecto a la correlación entre número de alumnos por aula y el apoyo emocional del profesorado, la prueba de $r$ de Pearson refleja un valor $r=.104$, con una significación $p=0.399>\alpha=.05$, por lo que esta relación ligeramente positiva y directa entre ambas variables no es significativa.

Respecto a los análisis de contraste de diferencias de medias con las otras dos variables, en la tabla 3 se reflejan los resultados obtenidos mediante el ANOVA de un factor a través del estadístico F de Snedecor:

Tabla 3

Resultados obtenidos con el ANOVA de un factor mediante F de Snedecor

\begin{tabular}{lccccc}
\hline \multicolumn{1}{c}{ Variables } & $\begin{array}{c}\text { Suma de } \\
\text { cuadrados }\end{array}$ & gl & $\begin{array}{c}\text { Media } \\
\text { de la raíz } \\
\text { cuadrática }\end{array}$ & $F$ & $p$ \\
\hline Titularidad del centro educativo & 18,066 & 29 & 0,623 & 0,992 & 0,503 \\
\hline $\begin{array}{l}\text { Docentes formados previamente en } \\
\text { formación socioemocional }\end{array}$ & 19,118 & 29 & 0,659 & 0,903 & 0,608 \\
\hline
\end{tabular}


Nota: La comparación de medias tiene una significación mayor a .05. Fuente: elaboración propia.

Como puede observarse en la tabla 3 del análisis ANOVA, en primer lugar, no existe una relación significativa entre la titularidad del centro educativo y la capacidad de gestión emocional del profesor para gestionar el clima de su aula, cuyo valor $\mathrm{F}=0,992$, le corresponde una sig. $=0,503>\alpha=0,005$. Por lo tanto, no existen diferencias en el procedimiento de los maestros para gestionar el clima emocional del aula en función de la titularidad del centro educativo. En segundo lugar, no existe una correlación relevante entre la capacidad de manejar el clima del aula de los profesores y el hecho de haber sido o no formados en inteligencia emocional, ya que su valor es $\mathrm{F}=0,903$, con una sig. $=0,608>\alpha=0,005$. Por consiguiente, no existen diferencias importantes entre los profesores que han sido formados y los que no para gestionar el clima de su clase.

\section{Conclusiones y discusión}

En primer lugar, en relación con el objetivo general de la investigación, se puede afirmar que, al contrario de lo que se podría esperar, no se percibe una clara relación entre la capacidad de los maestros para gestionar el clima emocional de su aula y su formación socioemocional, tal y como puede observarse en la prueba $t$ de Student (tabla 3). En la misma línea, se encuentran los resultados obtenidos por Cejudo y López-Delgado (2017). Sin embargo, Howes et al. (2008) y Little y Cohen-Vogel (2016) comprobaron que los profesores que habían sido instruidos en inteligencia emocional mostraban mayor eficacia en su forma de gestionar y apoyar el clima emocional de sus clases, facilitando así el desarrollo cognitivo, académico y emocional de sus estudiantes, además de propiciar mejores relaciones dentro de sus aulas.

Ulloa et al. (2016) también percibieron en su estudio experimental que los procedimientos utilizados por los docentes en sus cursos para generar un buen clima emocional y social están influidos por su formación y capacidad emocional. Asimismo, Gordillo et al. (2016) aportaron una experiencia de gran valor científico, puesto que comprobaron que cuando el profesorado cuenta con estrategias de gestión emocional adecuadas es capaz de 
solucionar de forma efectiva los problemas y conflictos generados en su aula.

En la misma línea, Curby, Brock y Hamre (2013) argumentan que los maestros que configuran un clima de aula positivo basado en el fomento de un buen respaldo emocional y social producen en sus estudiantes una reacción apropiada. Además, suelen sentirse más seguros en su capacidad para aprender. Otra importante conclusión es que, si los docentes instauran buenas relaciones con sus discentes, provocan a su vez interacciones correctas y provechosas entre ellos y con sus propios compañeros. Es decir, se sienten libres para poder expresar sus necesidades tanto a nivel académico como emocional (Ulloa et al., 2016).

Según Borrachero, Dávila, Costillo y Mellado (2017), los maestros que son más sensibles normalmente demuestran más entusiasmo, suelen utilizar palabras de reconocimiento de sus alumnos y les prestan apoyo emocional, establecen de forma clara las normas de la clase, están pendientes de las demandas académicas y emocionales de sus estudiantes, se preocupan por establecer relaciones adecuadas con sus alumnos y entre ellos y, con ello, suelen lograr mejores comportamientos en su alumnado y en sus deseos por aprender.

A su vez, Extremera, Rey y Pena (2016), afirman quelos maestros que tienen facilidad para regular sus emociones son más proclives a demostrar sentimientos positivos hacia sus alumnos. Además, los que son más tranquilos normalmente tratan a sus alumnos con más empatía e integran también a los que se comportan de forma más difícil y problemática.

En cuanto al objetivo propuesto de determinar el número de profesores que han sido formados o no en educación emocional, hemos comprobado que, de los 68 docentes, 28 -41,2\%- sí han recibido cursos de formación en sus centros; en cambio, 48 docentes $-58,8 \%-$ no los han recibido. Sin embargo, los docentes previamente formados en educación emocional no demuestran un mejor manejo emocional con sus alumnos, por lo que no parece ser una variable clave para la gestión del aula. En general, numerosos profesores sienten que tienen insuficientes capacidades para llevar a cabo su labor, a pesar de haber recibido una formación concreta y, por ello, solicitan cada vez más la formación oportuna que les dote 
de estrategias apropiadas para gestionar sus clases de manera más efectiva.

Pérez-Escoda et al. (2012) defienden la necesidad de instruir al profesorado emocionalmente para que pueda enseñar a sus alumnos desde la primera infancia habilidades interpersonales e intrapersonales. Asimismo, Cassullo \& García (2015) han demostrado que existe una relación positiva de las habilidades emocionales del profesor con la capacidad de los alumnos para interactuar entre ellos y con el propio docente, que favorece su motivación y autonomía para trabajar (Berrocal et al., 2017). Asimismo, la capacidad del profesor para crear un clima emocional adecuado favorece en sus alumnos el desarrollo de competencias emocionales y sociales - como la empatía y la asertividad- y fomenta en ellos la autonomía para la toma de decisiones y para realizar las actividades de clase, así como su capacidad para interactuar con los compañeros y favorecer un clima emocional positivo (Pérez-Escoda et al., 2012).

De acuerdo con Ulloa et al. (2016), los profesores necesitan tener formación en habilidades socioemocionales concretas que les ayuden a gestionar de forma positiva el clima de sus aulas, en las que prevalezcan, entre sus miembros, relaciones cálidas que promuevan la empatía, la asertividad, y a su vez, en las que existan unas líneas claras de actuación establecidas por el propio docente. García y Sánchez (2017) comprobaron que los profesores que tienen buena formación emocional suelen sentirse satisfechos con su gestión del aula porque crean interacciones adecuadas con sus alumnos y generan así un ambiente cálido y seguro que facilita el proceso de enseñanza-aprendizaje.

Asimismo, Pianta et al. (2014) perciben que los docentes no están actualizados en formación emocional y social y, por tanto, no cuentan con las herramientas y estrategias necesarias para ser emocionalmente inteligentes. Se hace necesaria, entonces, una instrucción socioemocional adecuada para dirigir y manejar el clima de su aula (Burrola-Herrera et al., 2016; Pérez-Escoda et al., 2012; Ros-Morente et al., 2017).

Con respecto al objetivo de conocer la relación entre la capacidad de los docentes para gestionar el clima de su aula y la titularidad del centro educativo, se ha verificado que no existe una vinculación 
evidente entre ambas, ya que el hecho de que se trate de un centro público, privado o concertado no incide directamente sobre la habilidad del maestro para crear un ambiente seguro y afectuoso que favorezca los procesos de aprendizaje de su alumnado.

Los resultados de esta investigación sobre la relación del número de alumnos y la forma de gestionar el clima del aula por parte de sus maestros son coincidentes con los del estudio de LoCasaleCrouch et al. (2007), ya que se ha apreciado que el número de alumnos por grupo de clase no condiciona la habilidad del maestro para gestionar el clima emocional y social de sus alumnos. Es decir, en algunas de las aulas en las que había más niños se observó que los profesores eran más habilidosos para establecer unas buenas relaciones con sus alumnos. En cambio, otros docentes que tenían menos alumnos en sus aulas, no presentaban una buena capacidad para generar un clima de interacciones apropiadas entre sus miembros. Sin embargo, autores como Lera (2007) o Barnett et al. (2010) en sus estudios percibieron todo lo contrario, es decir, que los profesores con una ratio menor se sentían más competentes a la hora de gestionar de forma más apropiada sus clases.

Una de las limitaciones encontradas se produjo en el proceso de recogida de datos para medir el clima de aula, debido a que, a través de la observación sistemática en algunas situaciones, hubo una variable extraña que pudo incidir de forma negativa en los resultados. Por ejemplo, al inicio de la observación, en las aulas los alumnos se comportaron de manera distinta con respecto a como suelen hacerlo en el día a día, lo cual pudo contaminar de manera muy sutil los resultados. Sin embargo, esta situación fue mejorando a medida que iba pasando el tiempo, ya que los estudiantes se sentían más tranquilos al acostumbrarse al proceso.

Por otro lado, los maestros también demostraron inquietud al inicio de la observación al sentirse contemplados por los observadores. A medida que pasó el tiempo, estuvieron más tranquilos y, por tanto, se empezaron a mostrar de forma más natural, lo cual facilitó la toma de datos para su interpretación y extraer unas conclusiones más realistas.

A través de la observación sistemática -técnica que puede conllevar cierta subjetividad por la actitud y el conocimiento de los observadores, pero que se solventó gracias a que la actividad fue 
realizada por dos supervisores-, se logró hacer una comparación que permitió bajar el nivel de subjetividad y modificar el grado de fiabilidad acerca del desarrollo del manejo del clima del aula.

Otra de las dificultades encontradas para la selección de la muestra fue la escasa colaboración por parte de muchos centros a los que se les escribió para acceder a sus aulas y así realizar la investigación. En algunas ocasiones se debió a que los padres no estaban de acuerdo por la ley de protección del menor y la de protección de datos, a pesar de haberles enviado una carta firmada en la que había un compromiso de confidencialidad y de anonimato de los datos; en otras, porque los profesores no querían participar por diversas razones, como incomodidad de ser observados y por no querer mostrar su forma de trabajar en el aula, entre otras. En otros centros educativos el rechazo fue inminente por parte de los directores, ya que no querían autorizar la entrada a sus aulas de personas ajenas que podían modificar el ritmo normal del proceso de enseñanza-aprendizaje.

En cuanto a los planteamientos de investigaciones futuras, se propone realizar un estudio de valoración sobre las competencias sociales y emocionales del propio profesorado y su capacidad para gestionar el clima de aula para conocer su propia apreciación de cómo lo hacen en sus clases y, de ese modo, realizar un estudio más amplio. Otra sugerencia es hacer una investigación de tipo experimental, en la que se configure un grupo experimental de docentes, que sean formados en competencias emocionales y sociales para manejar el clima del aula y otro de control, que no reciban ningún tipo de formación emocional -simplemente deben trabajar en sus clases de forma natural-, con la finalidad de evaluar si se dan diferencias entre ambos grupos en el manejo del clima de sus aulas. Por último, se propone seleccionar una muestra de la población de mayor tamaño y, a su vez, contar con más observadores-supervisores para la recogida de los datos de las clases que permitan hacer comparaciones entre ellos y que tengan mayor representatividad que faciliten su inferencia a la población. 


\section{Referencias}

Barnett, W., Epstein, D., Carolan, M., Fitzgerald, J., Ackerman, D., \& Friedman, A. (2010). The state of preschool 2010. Recuperado de https://eric.ed.gov/?id=ED582834

Berrocal, P., Cabello, R., \& Cobo, M. (2017). Avances en la investigación sobre competencias emocionales en educación. Revista interuniversitaria de formación del profesorado, (88), 15-26. Recuperado de: https:// scholar.google.es/scholar?hl=es\&as_sdt=0\%2C5\&q=Avances+en+la +investigaci\% $3 \%$ B3n+sobre+competencias+emocionales+en+educ aci\% $3 \%$ B3n\&btnG $=$

Bisquerra, R., \& Hernández-Paniello, S. (2017). Psicología positiva, educación emocional y el programa Aulas Felices. Papeles del Psicólogo, 38(1), 58-65. https://doi.org/10.23923/pap. psicol2017.2822

Bisquerra, R., \& Pérez-Escoda, N. (2007). Las competencias emocionales. Educación XXI: Revista de la Facultad de Educación, (10), 61-82. https://doi.org/10.5944/educxx1.1.10.297

Bisquerra, R., Pérez-González, J., \& García-Navarro, E. (2015). Inteligencia emocional en educación. Madrid: Síntesis.

Borrachero, A., Dávila, M., Costillo, E., \& Mellado, V. (2017). Las emociones del futuro profesorado de secundaria de ciencias y matemáticas, tras un programa de intervención. Ápice: Revista de Educación Científica, 1(1). https://doi.org/10.17979/arec.2017.1.1.2008

Burrola-Herrera, J., Burrola-Márquez, L., \& Viramontes-Anaya, E. (2016). Inteligencia emocional e integración grupal en el aula: dos consideraciones en la formación docente. Ra Ximhai, 12(6), 165-176. https://doi.org/10.35197/rx.12.01.e3.2016.09.jb

Caruana, A. (2013). Educación emocional en el aula. Documento presentado en el Congreso Internacional de Inteligencia Emocional y Bienestar. Zaragoza, España. Recuperado de http://www. congresointeligenciaemocional.com/

Cassullo, G., \& Livia-García, L. (2015). Estudio de las competencias socioemocionales y su relación con el afrontamiento en futuros profesores de Nivel Medio. Revista Electrónica Interuniversitaria de Formación del Profesorado, 18(1), 213-228. http://dx.doi. org/10.6018/reifop.18.1.193041

Cassullo, G., \& García, L. (2015). Estudio de las competencias socioemocionales y su relación con el afrontamiento en futuros profesores de Nivel Medio. Revista Electrónica Interuniversitaria 
de Formación del Profesorado, 18(1), 213-228. http://dx.doi. org/10.6018/reifop.18.1.193041

Cejudo, J., \& López-Delgado, M. (2017). Importancia de la inteligencia emocional en la práctica docente: un estudio con maestros. Psicología Educativa, 23(1), 29-36. https://doi.org/10.1016/j.pse.2016.11.001

Curby, T., Brock, L., \& Hamre, B. (2013). Teachers' emotional support consistency predicts children's achievement gains and social skills. Early Education y Development, 24(3), 292-309. https://doi. org/10.1080/10409289.2012.665760

Durlak, J. (2016). Programme implementation in social and emotional learning: basic issues and research findings. Cambridge Journal of Education, 46, 333-345. https://doi.org/10.1080/030576 $4 \mathrm{X} .2016 .1142504$

Extremera, N., Rey, L. \& Pena, M. (2016). Educadores de corazón. Inteligencia emocional como elemento clave en la labor docente. Padres y Maestros/Journal of Parents and Teachers, (368). https://doi.org/10.14422/pym.i368.y2016.011

García, M., \& Sánchez, L. (2017). El aprendizaje servicio y el desarrollo de las competencias emocionales en la formación inicial del profesorado. Contextos Educativos. Revista de Educación, 20, 127145. http://doi.org/10.18172/con.2991

Gordillo, M., \& Sánchez-Herrera, S. (2015). ¿Adquieren nuestros alumnos competencias emocionales en el grado de magisterio? Opción, 31(2), 532-556. Recuperado de https://www.redalyc.org/articulo. oa? $\mathrm{id}=31045568030$

Gordillo, M., Ruiz, M., Sánchez, S., \& Calzado, Z. (2016). Clima afectivo en el aula: vínculo emocional maestro-alumno. International Journal of Developmental and Educational Psychology, 1(1), 195-201. http:// dx.doi.org/10.1706o/ijodaep.2016.n1.v1.273

Howes, C., Burchinal, M., Pianta, R., Bryant, D., Early, D., Clifford, R. \& Barbarin, O. (2008). Ready to Learn? Children's Pre-Academic Achievement in Pre-Kindergarten Programs. Early Childhood Research Quarterly, 23(1), 27-50. https://doi.org/10.1016/j. ecresq.2008.08.001

Lera, M. (2007). Calidad de la educación infantil: Instrumentos de evaluación. Revista de Educación, 343, 301-323. Recuperado de: http://www.revistaeducacion.educacion.es/re343/re343_14.pdf

Little, M., \& Cohen-Vogel, L. (2016). Too Much Too Soon? An Analysis of the Discourses Used by Policy Advocates in the Debate over 
Kindergarten. Education Policy Analysis Archives/Archivos Analíticos de Políticas Educativas, 24, 1-35. http://dx.doi. org/10.14507/epaa.24.2293

LoCasale-Crouch, J., Konold, T., Pianta, R., Howes, C., Burchinal, M., Bryant, D., \& Barbarin, O. (2007). Observed Classroom Quality Profiles in State-Funded Pre-Kindergarten Programs and Associations with Teacher, Program, and Classroom Characteristics. Early Childhood Research Quarterly, 22(1), 3. https://doi.org/10.1016/j. ecresq.2006.05.001

Martínez, R., Rodríguez, B., Álvarez, L., \& Becedóniz, C. (2016). Evidence in promoting positive parenting through the Program-Guide to Develop Emotional Competences. Psychosocial Intervention, 25(2), 111-117. http://dx.doi.org/10.1016/j.psi.2016.04.001

Martínez-Izaguirre, M., Yániz, C., \& Villardón, L. (2017). Competencias profesionales del profesorado de educación obligatoria. Revista Iberoamericana de Educación, 74, 171192. Recuperado de: https://www.researchgate.net/profile/ Lourdes_Villardon/publication/319560166_Competencias_ Profesionales_del_Profesorado_de_Educacion_Obligatoria/ links/59b41d1c458515a5b49ob417/Competencias-Profesionalesdel-Profesorado-de-Educacion-Obligatoria.pdf

Pérez-Escoda, N., Filella, G., Alegre, A., \& Bisquerra, R. (2012). Desarrollo de la competencia emocional de maestros y alumnos en contextos escolares. Electronic Journal of Research in Educational Psychology, 10 (3), 1183-1208. https://doi.org/10.25115/ejrep. v10i28.1530

Pianta, R., Burchinal, M., Jamil, F., Sabol, T., Grimm, K., Hamre, B... \& Howes, C. (2014). A cross-lag analysis of longitudinal associations between preschool teachers' instructional support identification skills and observed behavior. Early Childhood Research Quarterly, 29, 144-154. https://doi.org/10.1016/j.ecresq.2013.11.006

Pianta, R., La Paro, K., \& Hamre, B. (2008). Classroom Assessment Scoring System, Pre-k. Baltimore, MD: Paul H. Brookes Publishing Co. Recuperado de https://search.proquest.com/openview/e402c58 033899ccdae4fa7370f3b2b29/1?pq-origsite $=$ gscholar\&cbl=27755

Retamal, J., \& González, S. (2019). De la microviolencia al clima escolar: claves de comprensión desde el discurso de profesores. Psicoperspectivas, 18(1), 25. http://dx.doi.org/10.5027/ psicoperspectivas-vol18-issue1-fulltext-1559 
Robira, A., \& Bris, M. (2012). Formación integral del profesorado: hacia el desarrollo de competencias personales y de valores en los docentes. Tendencias pedagógicas, 20, 51-70. Recuperado de: https://dialnet.unirioja.es/servlet/articulo? codigo $=4105055$

Ros-Morente, A., Filella, G., Ribes, R., \& Pérez-Escoda, N. (2017). Análisis de la relación entre competencias emocionales, autoestima, clima de aula, rendimiento académico y nivel de bienestar en Educación Primaria. Revista Española de Orientación y Psicopedagogía, 28(1), 8-18. https://doi.org/10.5944/reop.vol.28.num.1.2017.19355

Rueda, P., \& Filella, G. (2016). Importancia de la educación emocional en la formación inicial del profesorado. Intercambio/Échange, 1, 212219. https://doi.org/10.21001/ie.2016.1.17

Treviño, E. (2013). Calidad de la educación parvularia: las prácticas de clase y el camino a la mejora. Pensamiento Educativo. Revista de Investigación Educacional Latinoamericana, 5o(1), 40-62. https:// doi.org/10.7764/PEL.50.1.2013.4

Ulloa, M., Evans, I., \& Jones, L. C. (2016). The effects of emotional awareness training on teachers' ability to manage the emotions of preschool children: An experimental study. Escritos de psicología, 9(1), 1-14. https://doi.org/10.5231/psy.writ.2015.1711 